\title{
Magnetic phase diagrams from non-collinear canonical band theory
}

\author{
Shallcross, Sam; Nordstrom, L.; Sharma, S.
}

Published in:

Physical Review B Condensed Matter

Link to article, DOI:

10.1103/PhysRevB.76.054444

Publication date:

2007

Document Version

Publisher's PDF, also known as Version of record

Link back to DTU Orbit

Citation (APA):

Shallcross, S., Nordstrom, L., \& Sharma, S. (2007). Magnetic phase diagrams from non-collinear canonical band theory. Physical Review B Condensed Matter, 76(5), 054444. https://doi.org/10.1103/PhysRevB.76.054444

\section{General rights}

Copyright and moral rights for the publications made accessible in the public portal are retained by the authors and/or other copyright owners and it is a condition of accessing publications that users recognise and abide by the legal requirements associated with these rights.

- Users may download and print one copy of any publication from the public portal for the purpose of private study or research.

- You may not further distribute the material or use it for any profit-making activity or commercial gain

- You may freely distribute the URL identifying the publication in the public portal

If you believe that this document breaches copyright please contact us providing details, and we will remove access to the work immediately and investigate your claim 


\title{
Magnetic phase diagrams from non-collinear canonical band theory
}

\author{
S. Shallcross, ${ }^{1,2, *}$ L. Nordström, ${ }^{3}$ and S. Sharma ${ }^{4,5}$ \\ ${ }^{1}$ Lehrstuhl für Theoretische Festkörperphysik, Staudstrasse 7-B2, 91058 Erlangen, Germany \\ ${ }^{2}$ Department of Physics, Technical University of Denmark, Building 307, DK-2800 Kongens Lyngby, Denmark \\ ${ }^{3}$ Department of Physics, Condensed Matter Theory Group, Box 530, SE-751 21 Uppsala, Sweden \\ ${ }^{4}$ Fritz, Haber Institute of the Max Planck Society, Faradayweg 4-6, D-14195 Berlin-Dahlem, Germany \\ ${ }^{5}$ Institut für Theoretische Physik, Freie Universität Berlin, Arnimallee 14, D-14195 Berlin, Germany
}

(Received 24 March 2007; revised manuscript received 29 June 2007; published 24 August 2007)

\begin{abstract}
A canonical band theory of non-collinear magnetism is developed and applied to the close packed fcc and bcc crystal structures. This is a parameter-free theory where the crystal and magnetic symmetry and exchange splitting uniquely determine the electronic bands. In this way, we are able to construct phase diagrams of magnetic order for the fcc and bcc lattices. Several examples of non-collinear magnetism are seen to be canonical in origin, in particular, that of $\gamma$-Fe. In this approach, the determination of magnetic stability results solely from changes in kinetic energy due to spin hybridization, and on this basis we are able to analyze the microscopic reasons behind the occurrence of non-collinear magnetism in the elemental itinerant magnets.
\end{abstract}

DOI: 10.1103/PhysRevB.76.054444

PACS number(s): 75.10.-b

\section{INTRODUCTION}

Structural trends in the Periodic Table and their microscopic origin are now very well understood; in contrast, the situation for magnetic order is not so clear. This can be attributed both to the fact that only a subset of the Periodic Table is magnetic in the three dimensional solid-the Fe group and rare earths-and the much greater degree of freedom of magnetic ordering. Indeed, within the transition metal block (with the exception of Mn), all elements are found either as fcc, hcp, or bcc lattices, whereas a wealth of non-collinear magnetic structures may be found. For the transition metals near the center of the series, one typically finds antiferromagnetic (AFM) structures, while near the ends, ferromagnetism is favored. In between, one has several metals- $\gamma$ - $\mathrm{Fe}$ (fcc $\mathrm{Fe}$ ), bcc and fcc $\mathrm{Mn}$ - for which noncollinear structures can be found. ${ }^{1}$ On the other hand, the late rare earth group elements are non-collinear, while in the middle of the series, one has ferromagnetic Gd. ${ }^{2}$

This richness of magnetic structures means that most theoretical approaches focus on specific materials, and only a few attempts have been made to determine conditions wherein one places possible magnetic structures in some general scheme. A notable early attempt along such lines was the work of Pettifor ${ }^{3}$ who, on a similar basis to Friedel's theory for the stability of crystal structures, formulated a magnetic phase diagram in terms of the Stoner parameter to the bandwidth $I / W$ and the $d$-band occupation number. In this theory, the ferromagnetic and disordered local moment structures were considered, and the phase diagram revealed a simple argument as to why bcc $\mathrm{Fe}$ is a good local moment system, but fcc Co and fcc Ni are not. Heine and Samson, ${ }^{4}$ again using arguments based on generalized Stoner criteria, constructed a similar phase diagram but included also the AFM structure. They further made actual calculations of the generalized Stoner $I$ within the tight binding approximation, showing how $\gamma$-Fe was placed at the crossing point of the AFM and ferromagnetic (FM) stability criteria. Hirai, ${ }^{5}$ on the basis of an approximation to Hartree-Fock theory, calculated the energy of FM, AFM, and helical spin spiral structures as a function of an intra-atomic parameter and the $d$-band occupation number. The resulting phase diagram showed the appearance of a region of helical stability in between regions of FM and AFM stability.

Recently, an attempt was made to reexamine the issue of non-collinear stability on the basis of first principles calculations. ${ }^{6}$ Interestingly, it was found that materials collinear at their equilibrium moment were non-collinear for smaller (local) moments, induced either by pressure or a fixed spin moment procedure. On this basis, it was suggested both that any magnetic material could be made non-collinear under appropriate conditions and also that the primary factor governing the instability of the FM state toward a noncollinear one was the hybridization of crossing spin-up and spin-down bands at the Fermi level. The former suggestion is, at first sight, somewhat at variance with earlier work, particularly that of Hirai, where the occurrence of noncollinear magnetism was seen to be directly related to $d$-band filling.

It has been shown in the past that Andersen's canonical $d$-band theory gives a good qualitative account of crystal stability in the transition metal block and rare earths, ${ }^{7,8}$ even including the impact of collinear magnetism. ${ }^{9}$ In this theory, the canonical $d$ bands are determined only by the symmetry of the crystal lattice. Thus, by varying the $d$-band filling one obtains, from differences in the band energy between various crystal structures, the observed trends in crystal stability, i.e., $\mathrm{hcp} \rightarrow \mathrm{bcc} \rightarrow \mathrm{hcp} \rightarrow \mathrm{fcc}$ in a non-magnetic $d$ row. In the much more complicated case of non-collinear magnetism, such a parameter-free and conceptually simple theory which nevertheless yields the correct trends would be even more useful, particularly in light of the above discussion.

In the present work, we will show how canonical $d$-band theory, suitably generalized to deal with non-collinear magnetism, may be used to construct phase diagrams of magnetic order as well as explore their precise microscopic, i.e., band structure origins. In a way completely analogous to the usual canonical theory, the electronic bands are determined only by the crystal and magnetic symmetry and the exchange split- 
ting. The resulting phase diagrams can be considered as a "magnetic map of the $d$ band." With this in hand, we shall be able to clarify the role of spin hybridization in non-collinear stability and the apparent contradiction between Refs. 5 and 6.

It should be noted that a $d$-band only theory of noncollinear magnetism might be expected to be useful, given that in the Fe group the magnetic moment is dominated by electrons of $d$ character. However, the effect of $s d$ hybridization will, for instance, change the position of specific $d$ bands, and the physics of this will be absent in this approach. Thus, a non-collinear canonical $d$-band theory may also be used to judge the impact of $s d$ hybridization on the formation of non-collinear magnetic structures.

The remainder of this paper is structured as follows. In Sec. II, we describe canonical band theory and its generalization to deal with non-collinear magnetism, followed in Sec. III by details pertinent to its implementation. In Sec. IV, we present non-collinear magnetic phase diagrams for the fcc and bcc lattices, and in Sec. V we discuss the microscopic reasons for the structure of the phase diagrams. Finally, in Sec. VI we conclude.

\section{NON-COLLINEAR CANONICAL BAND THEORY}

We begin by briefly summarizing the canonical band theory and its origins; for simplicity of presentation, we will consider only one atom in the crystal basis. Within the linear muffin tin orbital (LMTO) or Korringa-Kohn-Rostocker electronic structure method in the atomic sphere approximation (ASA), the electronic bands are given by the secular equation

$$
[\mathbf{P}(E)-\mathbf{S}(\mathbf{k})] \mathbf{a}_{i}^{\mathbf{k}}=0,
$$

where $\mathbf{k}$ is a reciprocal lattice vector in the first Brillouin zone (BZ) and $i$ a band index. In this equation, the potential function $\mathbf{P}(E)$ is a diagonal matrix only dependent on the $l$ quantum number and determined by the choice of atomic species, while the structure matrix $\mathbf{S}(\mathbf{k})$ depends only on the symmetry of the crystal.

Neglecting the off-diagonal $l^{\prime} l$ blocks of the structure matrix, which amounts to neglecting all hybridization effects between different angular momentum channels, leads to a set of pure $l$ canonical bands given by $P_{l i}=S_{l i}(\mathbf{k})$ unique for each crystal structure. In cases where the physics of the $d$ band is expected to play a leading role, one can further neglect all but the $l^{\prime}=l=2$ block, and by approximating the potential function by $P=(E-C) /\left(\mu s^{2}\right)$, one finds a simple eigenvalue problem for the canonical $d$ bands,

$$
\left[\left(\epsilon_{i}^{\mathbf{k}}-C\right) /\left(\mu s^{2}\right) \mathbf{1}-\mathbf{S}(\mathbf{k})\right] \mathbf{a}_{i}^{\mathbf{k}}=0,
$$

where $1 \leq i \leq 5$ is a $d$-band index. Here, $C$ is an irrelevant parameter which simply sets the origin of the energy axis, while $\mu s^{2}$ transforms the canonical eigenvalues to a physical energy scale ( $\mu$ is the intrinsic band mass and $s$ the WignerSeitz radius). This latter parameter thus sets the $d$-band width. Since energy differences are unaffected by the choice of this parameter, we have, effectively, a parameter-free theory.
To develop a non-collinear magnetic generalization, one must first specify a particular class of magnetic structures. It is convenient to consider helical spin spiral structures since these contain both the FM and AFM structures as limits. In a helical spin spiral structure, the magnetization is given by

$$
m\left(\mathbf{R}_{n}\right)=\left(\sin \theta \cos \left(\mathbf{q} \cdot \mathbf{R}_{n}\right), \sin \theta \sin \left(\mathbf{q} \cdot \mathbf{R}_{n}\right), \cos \theta\right),
$$

with $\mathbf{R}_{n}$ a direct lattice vector and $\mathbf{q}$ a reciprocal lattice vector in the first BZ. The FM limit is found for $\mathbf{q} \rightarrow \mathbf{0}$ and AFM limits for $\theta=\pi / 2$ and certain high symmetry points on the BZ boundary. For instance, the $X(H)$ and $L(P)$ points in the fcc (bcc) BZ generate antiferromagnetic structures in the [001] and [111] directions, respectively.

In general, structures given by Eq. (3) will be incommensurate with the underlying lattice. They will, however, be invariant under a lattice translation $\mathbf{R}_{n}$ and a rotation about the spiral axis by $\mathbf{q} \cdot \mathbf{R}_{n}$, symmetry operations which form the so called spin-space group. ${ }^{10}$ This leads to a generalized Bloch theorem and an LMTO-ASA secular equation as before, ${ }^{11}$

$$
[\mathbf{P}(E)-\mathbf{S}(\mathbf{k}, \mathbf{q})] \mathbf{a}_{i}^{\mathbf{k}}=0,
$$

where the potential function $\mathbf{P}(E)$ and eigenvector $\mathbf{a}_{i}^{\mathbf{k}}$ are in the local frame of the spin spiral, that is, in the frame which at site $\mathbf{R}_{n}$ is rotated with respect to the global reference frame by the polar angles $\theta$ and $\phi=\mathbf{q} \cdot \mathbf{R}_{n}$. The potential function will thus be diagonal in spin space, while the structure matrix $\mathbf{S}(\mathbf{k}, \mathbf{q})$ given by

$$
\mathbf{S}(\mathbf{k}, \mathbf{q})=\left(\begin{array}{cc}
\mathbf{S}^{+}-\mathbf{S}^{-} \cos \theta & \mathbf{S}^{-} \sin \theta \\
\mathbf{S}^{-} \sin \theta & \mathbf{S}^{+}+\mathbf{S}^{-} \cos \theta
\end{array}\right),
$$

with

$$
S_{m^{\prime} m}^{ \pm}=\frac{1}{2}\left(S_{m^{\prime} m}^{\mathbf{k}+\mathbf{q} / 2} \pm S_{m^{\prime} m}^{\mathbf{k}-\mathbf{q} / 2}\right),
$$

has off-diagonal elements in spin space. As before, we now neglect all but the $l^{\prime}=l=2$ blocks in Eq. (4), and with the linear approximation for the potential function, which now becomes

$$
\mathbf{P}=(C-E) /\left(\mu s^{2}\right) \mathbf{1}+\left(\begin{array}{cc}
-\Delta / 2 & 0 \\
0 & \Delta / 2
\end{array}\right)
$$

( $\Delta$ is the exchange splitting), one finds an eigenvalue problem for the canonical bands of the spin spiral given by

$$
\left\{\left(\epsilon_{i}^{\mathbf{k}}-C\right) /\left(\mu s^{2}\right) \mathbf{1}-[\mathbf{S}(\mathbf{k})+\Delta]\right\} \mathbf{a}_{i}^{\mathbf{k}}=0 .
$$

Just as Eq. (2) determines a set of bands unique for each crystal symmetry, so does Eq. (8) for each symmetry of the spin-space group and a given exchange splitting. The eigenvectors and eigenvalues of Eq. (8) readily yield the density of states of each spin channel,

$$
D_{\sigma}(\epsilon)=\frac{1}{V_{B Z}} \int d \mathbf{k} \delta\left(\epsilon_{i}^{\mathbf{k}}-\epsilon\right) n_{\sigma i}^{\mathbf{k}},
$$

where $n_{\uparrow i}^{\mathbf{k}}\left(n_{\downarrow i}^{\mathbf{k}}\right)$ is the sum of up (down) squared components of the eigenvector $\mathbf{a}_{i}^{k}$. From this, the basic quantities of the 
theory can be derived; the electron number, magnetic moment, and one-electron energy, given by

$$
\begin{gathered}
n\left(E_{F}, \Delta\right)=\sum_{\sigma} \int^{E_{F}} d \epsilon D_{\sigma}(\epsilon), \\
m\left(E_{F}, \Delta\right)=\int^{E_{F}} d \epsilon\left[D_{\uparrow}(\epsilon)-D_{\downarrow}(\epsilon)\right], \\
e\left(E_{F}, \Delta\right)=\sum_{\sigma} \int^{E_{F}} d \epsilon \epsilon D_{\sigma}(\epsilon) .
\end{gathered}
$$

The natural parameters of the theory are thus the Fermi level $E_{F}$ and the exchange splitting $\Delta$; however, the physical parameters are the $d$-band filling and magnetic moment. Equations (10) and (11) must be solved to yield $E_{F}(n, m)$ and $\Delta(n, m)$, and hence $e\left(E_{F}(n, m), \Delta(n, m)\right) \equiv e(n, m)$.

In this approach, there is, of course, no intrinsic force causing the magnetization; it is instead imposed by setting the exchange splitting to a fixed value which thus plays the role of an external field. The energy contribution of this external field is included in the one-electron energy and so must be subtracted from it. Implicit in the atomic sphere approximation from which the canonical band theory is derived is that the magnetization in each sphere is given by $m \hat{\mathbf{z}}$ and is parallel to the field-in our case the external field-driving the exchange splitting. The field energy per site is thus given by

$$
E_{\text {ext }}=-m B_{e x t} .
$$

Since $B_{\text {ext }}=\Delta / 2$, we immediately find the kinetic energy of the canonical bands as

$$
T(n, m)=e(n, m)+\frac{1}{2} \Delta m .
$$

To the canonical kinetic energy can then be added a Stoner term modeling just the kind of intrinsic force that is missing,

$$
E(n, m)=-\frac{1}{4} \operatorname{Im}^{2}+T(n, m),
$$

resulting in a canonical Stoner theory. At this stage, one can see that there are a few choices for the physical variables of the theory. A fixed spin approach can be taken by considering different spin spirals for fixed values of $n$ and $m$, while to model the physics of the Fe-group magnets, the natural variables would be $n$ and the Stoner $I$. On the other hand, to model the rare earths, where the localized $f$ electrons impose an exchange field on the valence $s p d$, the natural choice would be $n$ and the exchange splitting $\Delta$.

As in the non-magnetic canonical band theory, a number of simple analytical results can be found for the noncollinear canonical bands. In particular, we mention that the center of gravity of each spin channel, defined as

$$
C_{\sigma}=\frac{\int_{-\infty}^{\infty} d \epsilon \epsilon D_{\sigma}(\epsilon)}{\int_{-\infty}^{\infty} d \epsilon D_{\sigma}(\epsilon)},
$$

may be shown from Eq. (8) to be given by

$$
C_{\sigma}=C+\sigma \frac{\Delta}{2},
$$

which is the non-collinear generalization of the fact that the center of gravity of a canonical band is zero. Of course, for the collinear state, this is trivial since the bands are rigidly shifted.

\section{COMPUTATIONAL DETAILS}

In order to solve Eqs. (8)-(12), we use a Monkhorst-Pack mesh with $18910,109800,216000,216000$, and 37820 k-points in the irreducible Brillouin zone for the FM structure, and spirals along $\Gamma X, X W, W L$, and $L G$, respectively. For the bcc irreducible Brillouin zone, we use 5200, 28 830, and $109800 \mathbf{k}$ points for the FM structure and spirals along $\Gamma \mathrm{H}$ and $H P$, respectively. The $\mathbf{k}$ integration is performed using an eigenvalue broadening of $4.5 \mathrm{mRy}$. For the potential parameters which, for the canonical $d$-band theory, simply set the origin and scale of energy, we take $C$ and $1 /\left(\mu s^{2}\right)$ from the corresponding values in non-magnetic fcc Fe. Since $1 /\left(\mu s^{2}\right)$ changes with electron number, this choice does not represent a physical scale (excepting near $n=7.0$ ), only a more convenient scale in comparison to the bare canonical units. We should stress that energy differences at a given band filling, our concern here, are unaffected by this choice.

In order to solve Eqs. (10) and (11), we minimize the function

$$
f=\left[n^{\prime}\left(E_{F}, \Delta, \mathbf{q}\right)-n\right]^{2}+\left[m^{\prime}\left(E_{F}, \Delta, \mathbf{q}\right)-m\right]^{2} .
$$

To facilitate this, it is convenient to precalculate the functions $n^{\prime}$ and $m^{\prime}$ and also the corresponding one-electron energy function $e^{\prime}\left(E_{F}, \Delta, \mathbf{q}\right)$ over the full range of $E_{F}$ and $\Delta$ and interpolate using cubic splines. By using an exponential mesh for the spline end points, we find that, over the whole range of $E_{F}$ and $\Delta$, the average error between the spline interpolation and direct integration is in the sixth significant figure, i.e., negligible. In this way, one can very rapidly minimize Eq. (18), whereas if $n^{\prime}$ and $m^{\prime}$ are repeatedly calculated by solving Eq. (8) over the Brillouin zone, this would be a very slow, and in fact impractical, procedure.

\section{CANONICAL PHASE DIAGRAMS}

For the calculation of canonical phase diagrams, we have chosen the variables to be the band filling $n$ and moment $m$. By doing so, we hope to arrive at an overall view of the role of the band term in non-collinear stability. In order to minimize the energy at each $(n, m)$, a particular set of spin spirals must be specified. One could, in principle, minimize with respect to all $\mathbf{q}$ and $\theta$ degrees of freedom in Eq. (3); however, 


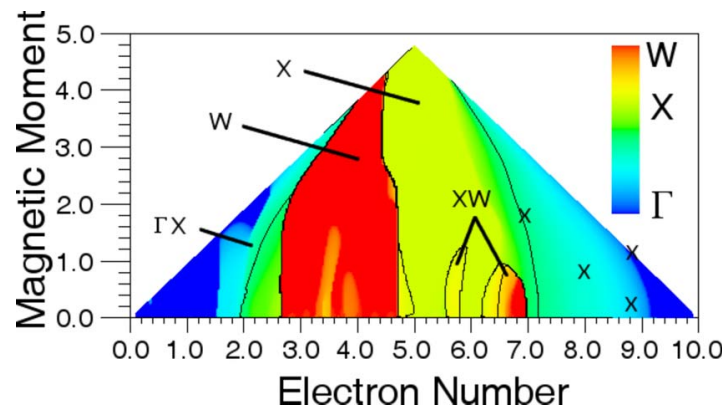

FIG. 1. (Color online) Canonical phase diagram for $\Gamma X W$ spin spirals in the fcc structure. Indicated by symbols are the regions of stability of different spiral structures. The lines beginning at $(n, m)=(7.2,0.0)$ and $(n, m)=(2.0,0.0)$ indicate the stability of the spiral $\mathbf{q}_{\Gamma X}=[0,0,0.5]$. The smaller size $X$ 's indicate points discussed in the text.

we have restricted ourselves to planar spin spirals, $\theta=\pi / 2$ in Eq. (3), generated by $\mathbf{q}$ vectors along the $\Gamma X W$ and $\Gamma H P$ lines of the fcc and bcc BZ's, respectively. The choice of planar spin spirals is convenient since, as was mentioned in Sec. II, this parameter space includes both the FM and AFM collinear limits as well as non-collinear states intermediate between them. Furthermore, there are sufficient experimental and theoretical data for a meaningful comparison with the canonical phase diagrams even with this restriction. For brevity, in the remainder of this paper, we shall refer to spin spirals with the $\mathbf{q}$ vector along an $A B$ symmetry line as $A B$ spin spirals.

In Figs. 1 and 2, it can be seen that, as expected, near half filling of the $d$-band AFM structures are found, while for the nearly fully occupied or empty $d$ band, FM structure is found. In both phase diagrams, it may be observed that the region of FM stability is too small. For instance, in the fcc phase diagram ferromagnetism becomes the stable solution for $n$ between 8.0 and $9.0 d$ electrons, whereas since fcc Co is FM and $\gamma$-Fe non-collinear, the boundary should lie between $n=7.0$ and 8.0.

We now consider the fcc phase diagram in more detail and its relation to known examples of non-collinearity. We

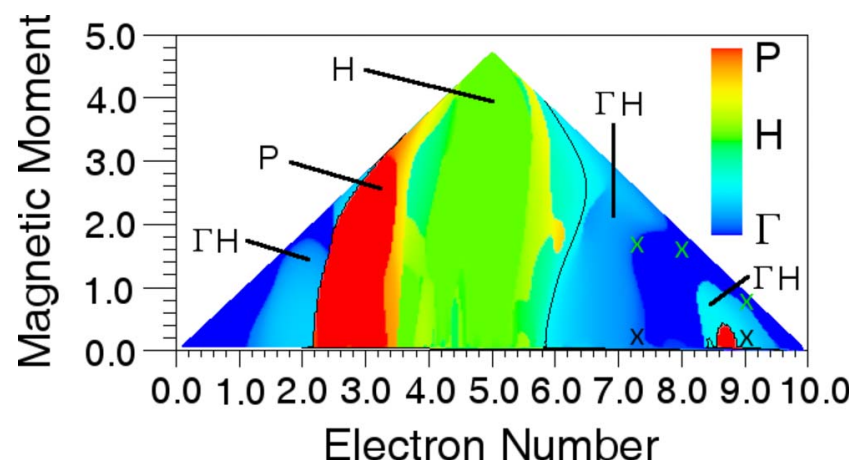

FIG. 2. (Color online) Canonical phase diagram for $Г H P$ spin spirals in the bcc structure. Indicated by symbols are the regions of stability of different spiral structures. The line beginning at $(n, m)=(5.8,0.0)$ indicates the stability of the spiral $\mathbf{q}_{\Gamma X}$ $=[0,0,0.5]$. X's indicate points discussed in the text. first discuss these examples. Recently, Ni, Co, and Mn were found to form spin spirals along the $\Gamma X$ direction when their moments were reduced to $0.2 \mu_{B}\left(\mathbf{q}_{\Gamma X}=[0,0,0.24]\right), 0.8 \mu_{B}$ $\left(\mathbf{q}_{\Gamma X}=[0,0,0.5]\right), \quad$ and $\quad 1.0 \mu_{B} \quad\left(\mathbf{q}_{\Gamma X}=[0,0,0.84]\right)$, respectively, ${ }^{6}$ with the spin spiral vector indicated in parentheses (we set $2 \pi / a=1$ ). The most well known example of non-collinearity is, however, $\gamma$-Fe. This is realized experimentally as precipitates grown in $\mathrm{Cu}$ and a spin spiral structure with $\mathbf{q}_{X W} \approx[0.0,0.2,1]$ is observed. ${ }^{12}$ Theoretically it is found that the magnetism is sensitive to all details of the calculation such as the electronic structure method, choice of exchange correlation functional, and lattice parameter. Recently, it was pointed out that, to a large extent, all this can be reduced to a dependence on the moment, which itself is very sensitive to lattice parameter and details of the calculation. ${ }^{13}$ With this in mind, we can give an approximate overview of the theoretical picture in $\gamma$-Fe by quoting what happens as the moment is changed. At small moments of $\approx 1.0 \mu_{B}$, nearly degenerate spin spirals with $\mathbf{q}_{\Gamma x}$ $\approx[0.0,0.0,0.8]$ and $\mathbf{q}_{X W} \approx[0.0,0.2,1]$ are found, with the latter having the lower energy. For moments of $\approx 2 \mu_{B}$, a deep minimum in the spin spiral energy at $\mathbf{q}_{\Gamma X}$ $\approx[0.0,0.0,0.5]$ is found. Finally, at moments of $\approx 2.5 \mu_{B}$, the FM structure is stabilized.

Most of these examples of non-collinearity can be found in Fig. 1. In order to compare with the data discussed above, the $d$ electron number corresponding to each element must be determined. One could use the $d$ electron number from self-consistent calculations- which yields for $\mathrm{Fe}, \mathrm{Co}$, and $\mathrm{Ni}$ values of 6.6, 7.5, and 8.6, respectively-or simply take the unhybridized $d$ electron numbers of 7, 8, and 9. Since we are not expecting quantitative results, this perhaps does not matter much, and so we will simply take $d$ electron numbers near the unhybridized integer values to correspond to the elements.

At a $d$ electron number of 8.8 , one finds ferromagnetism for a moment of $1.1 \mu_{B}$, while at $m=0.2 \mu_{B}$ a spin spiral with $\mathbf{q}_{\Gamma X}=[0,0,0.28]$, a behavior strikingly similar to that seen for fcc Ni. ${ }^{6}$ These points are indicated in Fig. 1, while the spin spiral spectrums are shown in panels (a) and (b) of Fig. 3 . On the other hand, for a $d$ electron number of 8.0, one finds for $m=0.8 \mu_{B}$ a spin spiral with $\mathbf{q}_{\Gamma X}=[0,0,0.46]$, similar to the behavior of fcc Co. ${ }^{6}$ This point is indicated in Fig. 1, with the spin spiral spectrum shown in panel (c) of Fig. 3. For a $d$ electron number of 7.0 and a moment of $1.8 \mu_{B}$ (again indicated by a cross in Fig. 1), one finds a deep energy minimum at $\mathbf{q}_{\Gamma X}=[0,0,0.60]$, which is similar to the intermediate moment spin spiral found in self-consistent calculations of $\gamma$-Fe [see panel (d) Fig. 3 for the corresponding spin spiral spectrum]. Interestingly, in Fig. 1 at band fillings $n$ $=6.25-7.0$ and moments up to $\approx 0.9 \mu_{B}$ (this is indicated by the boundary line in the corresponding region of Fig. 1), a small region of stability of $X W$ spin spirals and the $W$ point is found. A closer look at the spin spiral spectrum for $n$ $=6.5$ and $m=0.8$ [see panel (f) of Fig. 3] reveals a behavior very similar to that found for low moment $\gamma$-Fe in that one has nearly degenerate spiral minima at $\mathbf{q}_{\Gamma X}=[0,0,0.8]$ and $\mathbf{q}_{x W}=[0,0.32,1]$, with the latter having a marginally lower energy. It has been noted in the past ${ }^{4,5}$ that, in magnetic 


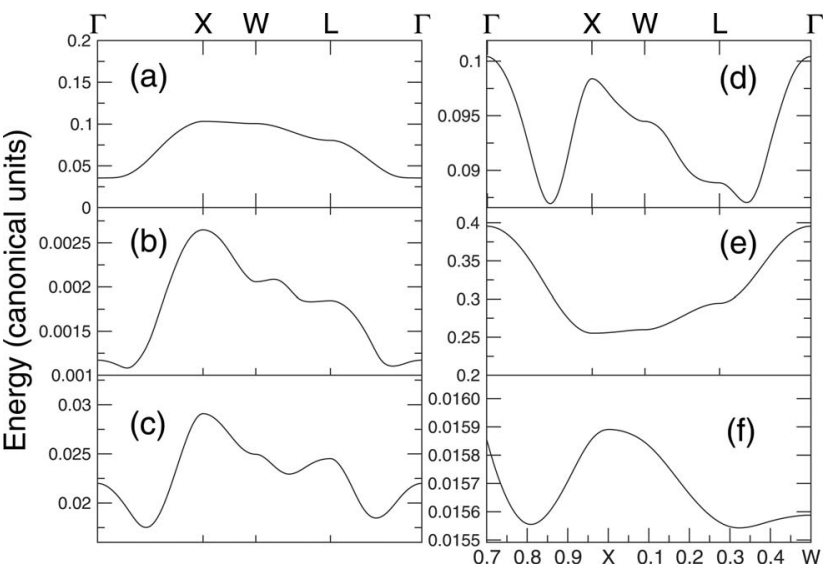

FIG. 3. Canonical spin spiral spectra for the symmetry path $\Gamma X W L \Gamma$ in the fcc Brillouin zone. Shown are spectra for (a) $n$ $=8.8, m=1.1$, (b) $n=8.8, m=0.2$, (c) $n=8.0, m=0.8$, (d) $n=7.0, m$ $=1.8$, (e) $n=5.0, m=3.3$, and (f) the region around the $X$ point for $n=6.5, m=0.8$. The top scale refers to graphs (a)-(e), and the bottom right to graph (f).

phase diagrams of the fcc lattice, $\gamma$-Fe appears at the crossing point of AFM to FM behavior. Here, we see a more detailed picture emerging, indicating that the non-collinear magnetism of $\gamma$-Fe is essentially canonical in origin. A number of obvious defects of the phase diagram in Fig. 1 must be pointed out. As has been mentioned, the region of stability of the FM phase is too small. A consequence of this is that a high-spin FM state is not found for electron numbers $n$ $=7.0-8.5$. Finally, missing in Fig. 1 is the $\Gamma X$ spiral found for $\mathrm{Mn}$ at a moment of $1.0 \mu_{B}{ }^{6}$

For the bcc lattice a similar situation holds, i.e., several examples of non-collinear magnetism from electronic structure calculations can be found, and hence are canonical in origin, but not all. Again, we briefly review the pertinent facts. Firstly, unlike fcc Co, bcc Co does not form a spin spiral except for very small moments, of the order of $0.15 \mu_{B} .{ }^{6}$ On the other hand bcc $\mathrm{Ni}$, at a moment of $0.2 \mu_{B}$, was found to form a spin spiral with $\mathbf{q}_{\Gamma X}=[0,0,0.18]$, and $\mathrm{Fe}$ and $\mathrm{Mn}$ for compressed volumes were found to form spin spirals with $\mathbf{q}_{\Gamma X}=[0,0,0.03]$ and $\mathbf{q}_{\Gamma X}=[0,0,0.20]$, respectively. ${ }^{6}$ Turning to the canonical bcc phase diagram, one finds that for a $d$ electron number of $9.0(\mathrm{Ni})$, one has ferromagnetism for $m=0.75 \mu_{B}$, while for a moment of $0.2 \mu_{B}$, one finds a spin spiral with $\mathbf{q}_{\Gamma X}=[0,0,0.36]$. Moreover, for $n=8.0(\mathrm{Co})$, there is a FM solution for $m=1.6 \mu_{B}$ and this is stable down to the smallest moments $(m$ $\left.=0.05 \mu_{B}\right)$, although there several almost degenerate spin spiral minima along the $\Gamma H$ and $H P$ symmetry lines. At a $d$ electron number of $7.3(\mathrm{Fe})$, one for $m=0.2 \mu_{B}$ finds a spin spiral with $\mathbf{q}_{\Gamma X}=[0,0,0.06]$, while for higher moments, $m$ $=1.7 \mu_{B}$, a FM solution is found. All this is strikingly similar to the results from electronic structure calculations. ${ }^{6}$ A further interesting observation is that, as it should be, the FM phase is more resistant to spin spiral formation for the bcc lattice as compared to the fcc. Also, the form of the boundary is different; near band fillings of $n=6.0$, the stability of the FM state is increased for smaller moments. This actually corresponds to what is seen in electronic structure calculations, ${ }^{1}$ fcc $\mathrm{Mn}$ is AFM, while bec Mn has a low-spin FM state which, as the moment is increased by increasing volume, leads through a non-collinear phase to a high-spin AFM state. ${ }^{1}$ On the other hand, the low-spin FM state near $n=6.0$ is not found in Fig. 2; it is unstable with respect to small q spin spirals. Furthermore, between $d$ electron numbers of 6.0 and 7.0, one can note a phase of non-collinear stability for high moments, clearly an artifact of the canonical theory.

We now discuss some of the more general features of the canonical phase diagrams. Two things stand clear from the preceding discussion. Firstly, in agreement with Ref. 6, one cannot consider non-collinearity to be an "exotic" feature of itinerant magnetic materials. In these phase diagrams, on the contrary, for large ranges of $d$ electron numbers and moments, non-collinear structures are more stable than collinear ones. However, rather than this non-collinearity being arbitrary, in the sense of Ref. 6 that any material could be made non-collinear under appropriate conditions, it is deeply connected to the $d$-band electron number. It is not quite the case that regions of non-collinear stability are always to be found between those of the AFM and FM structures, as was found in the work of Hirai. ${ }^{5}$ The low-spin incursion of $\Gamma H$ structure into ferromagnetism for $n \approx 8.5$ (low-spin bcc $\mathrm{Ni}$ ) and the appearance of the $X W$ phase at the boundary of the AFM-1 and $\Gamma X$ phases (low-spin $\gamma-\mathrm{Fe}$ ) are counterexamples to this general trend. Other examples can be seen in Figs. 1 and 2. Finally, the phase diagrams do help give a different perspective on the small moment non-collinearity in fcc $\mathrm{Ni}$ and fcc Co. In these cases, it can be seen that reducing the moment has precisely the same effect as reducing the $d$ electron number, simply bringing the material into the $\Gamma X$ region of stability.

\section{ROLE OF SPIN HYBRIDIZATION}

The fact that several features of the magnetic phase diagrams shown in the previous section correspond, qualitatively, to features seen either in experiment or electronic structure calculations for fixed spin moment makes it worthwhile to consider their microscopic origin. In the canonical approach there is, of course, no intrinsic contribution from the Coulomb energy of the electrons. The electron-electron interaction is instead bound up in the on-site Stoner term as the Stoner parameter to the $d$-band width $I / W$, which, in a fixed spin procedure, drops out of the energy expression [see Eq. (15)]. Since there is also no hybridization between different $l$ channels, the determination of magnetic structure is driven solely by spin hybridization, that is, hybridization effects between opposite spin channels of the $d$ band.

With this in mind, the key question to be answered is how spin hybridization brings about the observed trends in magnetic order. This is interesting since the role of spin hybridization in non-collinear magnetism has been emphasized recently in Ref. 6 and is also at the root of the well known collinear AFM $\rightarrow$ FM trend. ${ }^{1}$ We shall consider the fcc lattice as an example and specifically the change in magnetic order from $\mathrm{AFM}-1 \rightarrow \Gamma X \rightarrow \mathrm{FM}$ as the $d$ electron number is in- 


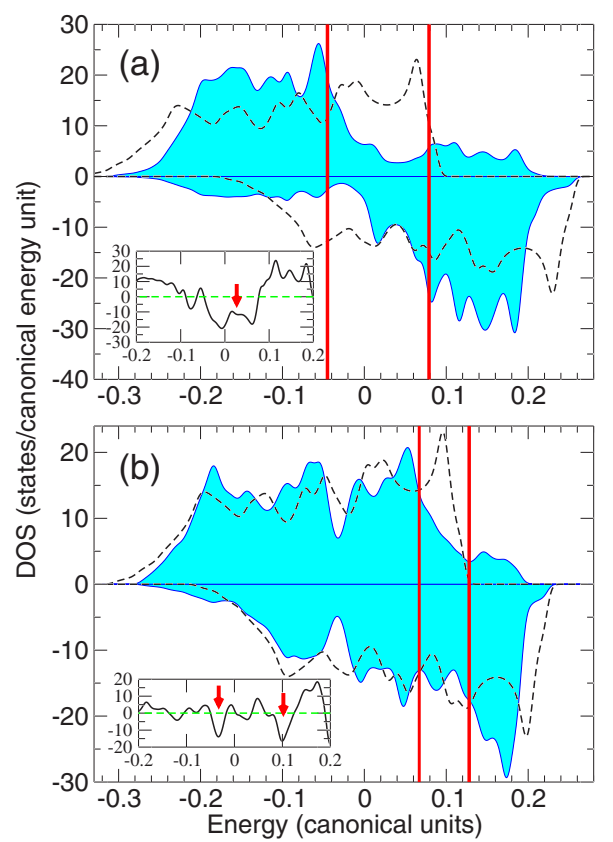

FIG. 4. (Color online) Canonical density of states (DOS) for (a) AFM-1 and (b) spin spiral $\mathbf{q}_{\Gamma X}=[0,0,0.5]$ structures. Broken line indicates the FM DOS for the same exchange splitting. In each case, the inset graph shows the difference between the AFM-1 or spiral DOS and the FM DOS. Vertical lines indicate a region where the total AFM-1 or spiral DOS is lower than the FM DOS.

creased from half-filling (see Fig. 1). In Fig. 4, we show the canonical density of states (DOS) for the AFM-1 structure [panel (a)] and the $\mathbf{q}_{\Gamma X}=[0,0,0.5]$ spin spiral [panel (b)]. In each case, the broken lines indicate the corresponding FM DOS. The exchange splitting is set to give $m=3.3$ at $n=5.0$ for the AFM-1 structure and $m=1.8$ at $n=7.0$ for the $\Gamma X$ spin spiral. The latter point in the phase diagram shows a behavior similar to the intermediate moment spin spiral found in $\gamma$-Fe [see panel (d) of Fig. 3]. What can clearly be seen is the creation of hybridization gaps in the DOS, which, for the AFM-1 structure consists of a single large gap at the center of the band and for the $\Gamma X$ structure several gaps, but principally, two at energies of +0.1 and -0.05 . This is brought out clearly in the inset graphs of Fig. 4 which show the difference between the AFM-1 or spiral DOS and that of the corresponding FM DOS, with the gaps indicated by arrows.

The effect of such hybridization gaps upon magnetic stability depends crucially on their relation with the Fermi energy. When the Fermi energy lies in a hybridization gap, the non-collinear (or AFM) structure will be energetically favorable as spectral weight in the majority (minority) channel is shifted to lower (higher) energies. On the other hand, if the Fermi level lies in either side of the hybridization gap, it will be in the "hybridization peak" and consequently the structure which gives rise to this DOS will be energetically unfavorable. It should be pointed out that such conclusions hold true also when one considers not the one-electron energy but the kinetic energy, which is the physically relevant quantity.

Given that the AFM-1 structure places a hybridization gap at $E=0$, while the $\mathbf{q}_{\Gamma X}=[0,0,0.5]$ structure places the gaps

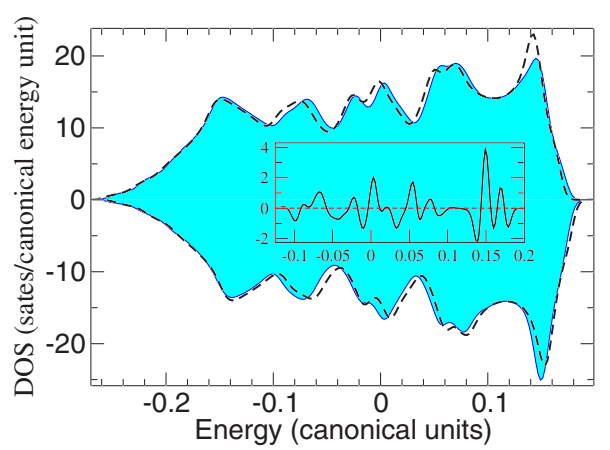

FIG. 5. (Color online) Canonical density of states (DOS) for the spin spiral $\mathbf{q}_{\Gamma X}=[0,0,0.3]$ structure and, indicated by the broken lines, the FM DOS with identical exchange splitting. The inset shows the difference between the $\mathbf{q}_{\Gamma X}=[0,0,0.3]$ and FM DOS.

off center at $E \approx-0.05$ and $E \approx 0.1$, an explanation of the AFM- $1 \rightarrow \Gamma X \rightarrow$ FM trend can be found. At half filling, the Fermi level is in the center of the $d$ band and hence placed in the very strong pseudogap evident in Fig. 4. The AFM-1 structure is thus stabilized. However, as the band filling is increased, the Fermi level enters the minority "upper hybridization peak" of the AFM-1 DOS. The kinetic energy can then be lowered by a change in structure to the $\Gamma X$ spin spiral since, although it has a weaker hybridization gap, it is placed off center at $E \approx 0.1$, and for higher band fillings this is where the Fermi energy lies. The band fillings corresponding to the gap in panel (b) of Fig. 4 are centered around $n=7.0$ (in fact, $n=6.75-8.50$ ), which confirms that this is the mechanism which stabilizes the non-collinear order found in intermediate moment $\gamma$-Fe. As $n$ increases further, the Fermi level will continuously be entering the upper hybridization peak of the DOS, driving the spiral $\mathbf{q}$ vector toward the $\Gamma$ point. In this way, for sufficiently large $n$, FM order is finally stabilized.

It is important to note that the pronounced hybridization gaps found in the spiral DOS for $\mathbf{q}$ vectors far from the $\Gamma$ point are not preserved as $\mathbf{q} \rightarrow \mathbf{0}$ or as $\Delta \rightarrow 0$. In Fig. 5, we show the DOS of the $\mathbf{q}_{\Gamma X}=[0,0,0.3]$ spiral, with the exchange splitting set to give a moment of $0.2 \mu_{B}$ at $n=8.8$. The latter point in the phase diagram shows a behavior similar to that of fixed low moment fcc Ni [see panel (b) Fig. 3, this point is also marked on the fcc phase diagram Fig. 1]. Although there are no clear hybridization gaps, the quantity $D_{\Gamma X}(\epsilon)-D_{F M}(\epsilon)$ (shown in the inset) reveals a behavior very similar to that seen in Fig. 4, although with considerably more structure. An inspection of the band structure, see Fig. 6, corresponding to the DOS presented in Figs. 4(a) and 5 reveals clearly the change that takes place as spin hybridization becomes weaker. In the former case, all bands shift significantly in energy, leading to a hybridization gap on an energy scale of the $d$-band width. In contrast, the band structure of the $\mathbf{q}_{\Gamma X}=[0,0,0.3]$ spiral considered in Fig. 5 shows recognizably the topology of the FM state, the most significant difference being level repulsions at intersections of spin-up and spin-down bands. This leads to local hybridization gaps on an energy scale much smaller than that of the $d$-band width, the presence of which is masked in the total 


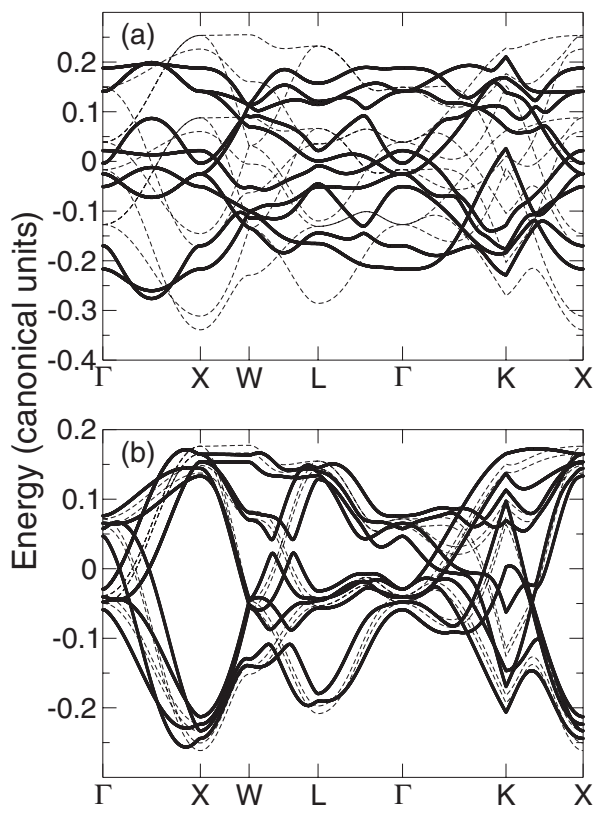

FIG. 6. Canonical band structure for the (a) high-spin AFM-1 and (b) low-spin $\mathbf{q}_{\Gamma X}=[0,0,0.3]$ spin spiral structures. In each case, the corresponding FM band structure is indicated by the broken lines.

DOS by states not involved in the hybridization.

Although this explanation is of a specific trend, it may readily be seen that the basic concept is more generally applicable, simply because such hybridization gaps will always be present in non-collinear, or AFM, structures. Trends in magnetic order thus have their microscopic origin in the relationship between the placement of spin hybridization gaps and the Fermi level. These gaps, however, exist on a variety of energy scales depending on the strength of the spin hybridization. The explanation presented here thus interpolates between the well known explanation for the stability of AFM structures at half filling ${ }^{1}$ and the more recent suggestion that it is local hybridization gaps at the Fermi level which play an important role in non-collinear magnetism. ${ }^{6}$

\section{CONCLUSIONS}

In this work, we have presented a non-collinear generalization of Andersen's canonical $d$-band theory, which has been used to generate phase diagrams of magnetic order for the fcc and bcc lattices. In large regions of these phase diagrams, non-collinear order is stabilized, and we have identified several examples of what might be called canonical noncollinear magnetism. These examples of non-collinearity are stabilized simply by the spin hybridization of the canonical $d$ band. In particular, the $\mathbf{q}=[0,0,0.5]$ and $\mathbf{q}=[0,0.2,1.0]$ spin spiral structures found in $\gamma$-Fe are canonical, with the former part of a general $\mathrm{AFM} \rightarrow \Gamma X \rightarrow \mathrm{FM}$ trend which includes also the low moment non-collinearity of fcc Co and Ni.

On the other hand, in some respects, the phase diagrams do not agree with what is found in electronic structure calculations. The most striking disagreement is that the region of FM stability is too small in both the fcc and bec phase diagrams. Since in the canonical $d$-band approach we have explicitly excluded $s d$ hybridization, it is tempting to conclude that such hybridization, in fact, favors the stability of the FM state over non-collinear states. This will be explored in a future work.

We have further analyzed the microscopic driving forces behind the structure of the canonical phase diagrams. In agreement with Ref. 6, we find that hybridization gaps brought about by spin mixing in the non-collinear, or AFM, states are found to be crucial. However, they may occur on a wide range of energy scales, from that of the $d$-band width to an energy scale where it is only states near the Fermi level which dominate. This is quite a natural conclusion since it unites the suggestion of Ref. 6 with the well known physics explaining the stability of AFM states near half filling.

\section{ACKNOWLEDGMENTS}

S. Shallcross is pleased to acknowledge stimulating conversations with $\mathrm{H}$. L. Skriver. S. Sharma would like to acknowledge NoE NANOQUANTA Network (NMP4-CT2004-50019) and Deutsche Forschungsgemeinschaft for financial support. *sam_shallcross@yahoo.co.uk

${ }^{1}$ J. Kübler, Theory of Itinerant Magnetism (Clarendon, Oxford, 2000).

${ }^{2}$ J. Jensen and A. R. Mackintosh, Rare Earth Magnetism (Clarendon, Oxford, 1991).

${ }^{3}$ D. G. Pettifor, J. Magn. Magn. Mater. 15-18, 847 (1980).

${ }^{4}$ V. Heine and J. H. Samson, J. Phys. F: Met. Phys. 13, 2155 (1983).

${ }^{5}$ K. Hirai, J. Phys. Soc. Jpn. 51, 1134 (1982).

${ }^{6}$ R. Lizárraga, L. Nordström, L. Bergqvist, A. Bergman, E. Sjöstedt, P. Mohn, and O. Eriksson, Phys. Rev. Lett. 93, 107205
(2004).

${ }^{7}$ O. K. Andersen and O. Jepsen, Physica B \& C 91, 317 (1977).

${ }^{8}$ H. L. Skriver, Phys. Rev. B 31, 1909 (1985).

${ }^{9}$ P. Söderlind, R. Ahuja, O. Eriksson, J. M. Wills, and B. Johansson, Phys. Rev. B 50, 5918 (1994).

${ }^{10}$ W. Brinkman and R. J. Elliot, J. Appl. Phys. 37, 1457 (1966).

${ }^{11}$ L. M. Sandratskii, Adv. Phys. 47, 91 (1998).

${ }^{12}$ T. Naono and Y. Tsunoda, J. Phys.: Condens. Matter 16, 7723 (2004).

${ }^{13}$ S. Shallcross, A. E. Kissavos, S. Sharma, and V. Meded, Phys. Rev. B 73, 104443 (2006). 